\title{
ON CONTINUOUS SPECTRUM OF THE LINEARISED WATER-WAVE PROBLEM IN BOUNDED DOMAINS
}

\author{
Jussi Martin \\ University of Helsinki, Department of Mathematics and Statistics \\ P.O. Box 68, FI-00014 Helsinki, Finland; jussi.martin@helsinki.fi
}

\begin{abstract}
We study the spectral problem of the linearised theory of water-waves, in a bounded domain with cuspidal edge. We show that the continuous spectrum of the problem is the set of non-negative real numbers, if the sharpness exponent is large.
\end{abstract}

\section{Introduction and the main result}

1.1. Preface. In the linearised theory of water-waves the wave solutions are described by velocity potentials which satisfy a mixed boundary value problem for the Laplace equation. In particular Steklov spectral boundary condition is posed on the horizontal water surface (see (1.2)-(1.4) below, and [19, 5, 6] for the physical background), and the spectral parameter is proportional to the frequency of the wave. In unbounded domains the continuous spectrum of this linearised water-wave problem is typically non-empty, a fact which is related to the existence of propagating waves.

In this paper we study the continuous spectrum of the linearised water-wave problem in bounded domains. Our domains will have cuspidal edge at the shoreline, which causes the continuous spectrum to appear. We show that if the sharpness exponent $m$ is greater than 2 , then the continuous spectrum is maximal in the sense that it consists of the whole set of non-negative real numbers.

Previously this problem has been studied in similar domains, but with different values of the sharpness exponent. In [16] it was shown that for $m<2$ the spectrum is discrete, and for two-dimensional water-wave problem the discreteness of the spectrum was proved for the first time in [3]. In the case $m=2$ the spectrum contains an interval $[c, \infty)$ for certain $c>0$. This result was proved in [8] and an analogous result for a domain with submerged object touching the water-surface at one point, was proved in [16] (see also the remarks at the end of this paper for further details).

1.2. Formulation of the problem. In the sequel we denote a point $x \in \mathbf{R}^{3}$ as $x=\left(y_{1}, y_{2}, z\right)$ in order to make a distinction between vertical and horizontal coordinates.

Let $\Lambda$ be a domain in the horizontal plane $\left\{x \in \mathbf{R}^{3}: z=0\right\}$, and let the closed simple $C^{2}$-contour $\gamma$ be the edge of $\Lambda$. Let also $\Sigma$ be a $C^{2}$-surface in the lower halfspace $\mathbf{R}_{-}^{3}=\left\{x \in \mathbf{R}^{3}: z<0\right\}$ with the same edge $\gamma$, and let $\Omega \subset \mathbf{R}^{3}$ be the interior domain bounded by $\Lambda \cup \Sigma \cup \gamma$.

doi:10.5186/aasfm.2013.3839

2010 Mathematics Subject Classification: Primary 35J20; Secondary 35P99, 76B15.

Key words: Linearised water-wave problem, continuous spectrum, cuspidal domain.

This research was supported by the Academy of Finland project "Functional analysis and applications". 
In the $\delta$-neighbourhood $\mathcal{U}_{\delta} \subset \Pi$ of the contour $\gamma \subset \Pi$ we introduce the natural system of the curvilinear coordinates $(\nu, \tau)$, where $\nu$ is the oriented distance to $\gamma$, $\nu>0$ inside $\Lambda$, and $\tau$ is the curve length on $\gamma$. We assume that in the vicinity of $\gamma \subset \mathbf{R}^{3}$ the domain $\Omega$ is given by the inequalities

$$
-\nu^{m} g(\nu, \tau)<z<0, \quad \nu>0,
$$

where $m>1$ and $g$ is a positive $C^{2}$-function on $[0, \delta] \times \gamma$.

In the domain $\Omega$ we consider the linearised water-wave problem

$$
\begin{aligned}
-\Delta_{x} \Phi(x) & =0, \quad x \in \Omega, \\
\partial_{n} \Phi(x) & =0, \quad x \in \Sigma, \\
\partial_{z} \Phi(x) & =\lambda \Phi(x), \quad x \in \Lambda .
\end{aligned}
$$

where $\Delta_{x}$ is the Laplacian, $\partial_{n}$ is the derivative along the outward normal $n$, and $\Phi$ denotes the velocity potential and $\lambda$ is the spectral parameter proportional to the square of the frequency of harmonic oscillations. The condition (1.4) is called a Steklov spectral boundary condition.

To give exact meaning to spectral concepts, such as continuous spectrum, we will formulate the problem (1.2)-(1.4) as a standard spectral problem for a certain operator $K_{\Omega}$.

1.3. The operator formulation of the problem. We define the Hilbert space $\mathcal{H}(\Omega ; \Lambda)$, as the completion of the space $C_{c}^{\infty}(\bar{\Omega} \backslash \gamma)$ of compactly supported smooth functions with respect to the norm $\|\Phi ; \mathcal{H}(\Omega ; \Lambda)\|:=\langle\Phi, \Phi\rangle_{\Omega}^{1 / 2}$, where

$$
\langle\Phi, \Psi\rangle_{\Omega}=\left(\nabla_{x} \Phi, \nabla_{x} \Psi\right)_{\Omega}+(\Phi, \Psi)_{\Lambda}
$$

and $(\cdot, \cdot)_{\Omega}$ and $(\cdot, \cdot)_{\Lambda}$ are the intrinsic inner products in the spaces $L^{2}(\Omega)$ and $L^{2}(\Lambda)$, respectively.

One particular property of the space $\mathcal{H}(\Omega ; \Lambda)$ is that the trace on $L^{2}(\Lambda)$, denoted by $\Psi(\cdot, 0)$, exists for all $\Psi \in \mathcal{H}(\Omega ; \Lambda)$. In the sequel the trace $\Psi(\cdot, 0)$ will be often abbreviated simply as $\Psi$.

If $\lambda>0$ and $m>1$, then a classical solution of (1.2)-(1.4) must vanish on $\gamma$ for (1.3) and (1.4) to hold simultaneously, since the $z$-direction is parallel to the normal direction of $\bar{\Sigma}$ on $\gamma$ if $m>1$. Also, the first Green formula implies that

$$
\left(\nabla_{x} \Phi, \nabla_{x} \Psi\right)_{\Omega}=\lambda(\Phi, \Psi)_{\Lambda}, \quad \Psi \in C_{c}^{\infty}(\bar{\Omega} \backslash \gamma),
$$

if $\Phi$ is a classical solution of the problem (1.2)-(1.4) and $\Psi \in C_{c}^{\infty}(\bar{\Omega} \backslash \gamma)$ is arbitrary. Thus it is natural to define a weak solution of (1.2)-(1.4) to be a function $\Phi \in \mathcal{H}(\Omega ; \Lambda)$ for which

$$
\left(\nabla_{x} \Phi, \nabla_{x} \Psi\right)_{\Omega}=\lambda(\Phi, \Psi)_{\Lambda} \quad \text { for all } \Psi \in \mathcal{H}(\Omega ; \Lambda) .
$$

This leads us to the operator formulation of the spectral problem (1.2)-(1.4).

Namely, we define the operator $K_{\Omega}$ from the space $\mathcal{H}(\Omega ; \Lambda)$ to itself by the formula

$$
\left\langle K_{\Omega} \Phi, \Psi\right\rangle_{\Omega}=(\Phi, \Psi)_{\Lambda}, \quad \Phi, \Psi \in \mathcal{H}(\Omega ; \Lambda) .
$$

This operator is symmetric, bounded and therefore self-adjoint. Adding the term $(\Phi, \Psi)_{\Lambda}$ to both sides of the equation (1.5) and multiplying them by $\mu=(1+\lambda)^{-1}$, we see that the problem (1.5) is equivalent to the equation

$$
K_{\Omega} \Phi=\mu \Phi, \quad \Phi \in \mathcal{H}(\Omega ; \Lambda) .
$$


The spectrum of the problem (1.2)-(1.4) and the spectrum of the operator $K_{\Omega}$ are now related in the following way. Let $\lambda \in \mathbf{C}$; then $\lambda$ is in the spectrum of the problem (1.2)-(1.4) if and only if $\mu=(1+\lambda)^{-1}$ is in the spectrum of $K_{\Omega}$. The same relation holds also for discrete, continuous and essential spectra.

Since the operator $K_{\Omega}$ is positive and $\left\|K_{\Omega}\right\| \leq 1$, its spectrum is real valued and contained in the interval $[0,1]$; thus the spectrum of the problem $(1.2)-(1.4)$ is contained in the interval $[0, \infty)$.

1.4. The main result. The definitions of continuous and essential spectra differ in different books, we use those introduced in [2]. Namely, the point spectrum of an operator $A$ is denoted by $\sigma_{p}(A)$, the continuous spectrum is the set

$$
\sigma_{c}(A)=\{\lambda \in \mathbf{C}: R(A-\lambda I) \neq \overline{R(A-\lambda I)}\}
$$

where $R$ denotes the range of given operator, and the essential spectrum is the set

$$
\sigma_{e}(A)=\sigma_{c}(A) \cup \sigma_{p}^{\infty}(A)
$$

where $\sigma_{p}^{\infty}(A)$ is the set of eigenvalues with infinite multiplicity. It should be noted, that with these definitions it is possible that $\sigma_{p}(A) \cap \sigma_{c}(A) \neq \varnothing$.

We are now ready to state our main result.

1.6. Theorem. If $m>2$, then the continuous spectrum of the problem (1.2)(1.4) constitutes the whole spectrum, and it is the half-line $[0, \infty)$.

\section{Preliminaries and outline of the proof}

2.1. Preliminaries. We now present shortly some concepts and theorems which will be used in the proof of Theorem 1.6.

2.1. Definition. A sequence $\left(\Psi_{j}\right)_{j=1}^{\infty}$ is called singular for the self-adjoint operator $A$ at a point $\lambda$, if the following conditions are satisfied:

i) $\inf _{j}\left\|\Psi_{j}\right\|>0, \quad$ ii) $\Psi_{j} \stackrel{w}{\rightarrow} 0, \quad$ iii $)\left(\Psi_{j}\right)_{j=1}^{\infty} \subset D(A), \quad$ iv $)(A-\lambda) \Psi_{j} \rightarrow 0$.

If $A$ is a self-adjoint operator, then the following two theorems are valid for $A$ (proofs of these theorems can be found from [2]).

2.2. Theorem. A point $\lambda$ belongs to $\sigma_{e}(A)$ if and only if there exists a singular sequence for $A$ at $\lambda$.

2.3. Theorem. $\sigma_{c}(A)$ is the set of non-isolated points of $\sigma(A)$.

2.2. Outline of the proof. We proceed in proving Theorem 1.6 by constructing a singular sequence at an arbitrary given point $\mu=(1+\lambda)^{-1} \in(0,1)$ for the operator $K_{\Omega}$, and then conclude by using Theorem 2.2 that $(0,1) \subset \sigma_{e}\left(K_{\Omega}\right)$. After this, since $\sigma\left(K_{\Omega}\right)$ is closed, Theorem 2.3 shows us that $[0,1] \subset \sigma_{c}\left(K_{\Omega}\right)$, which in turn implies that the continuous spectrum of the problem $(1.2)-(1.4)$ is the set $[0, \infty)$.

\section{Construction of the singular sequence}

3.1. Overview. In this chapter we construct a sequence $\left(\Psi_{j}\right)_{j=1}^{\infty} \subset \mathcal{H}(\Omega ; \Lambda)$, and prove it to be singular at a given point $\mu=(1+\lambda)^{-1} \in(0,1)$ for the operator $K_{\Omega}$. The construction will be done by first defining a sequence $\left(\Phi_{j}\right)_{j=1}^{\infty}$ of functions depending on the boundary distance coordinates $\nu$ and $\tau$. 
Concerning the notation of this paper, $C, C^{\prime}, c$ and so on denote positive constants which may vary from place to place and which are independent of the functions and variables in the given expressions; whit the index $j \in \mathbf{N}_{+}$also considered as a variable. We will also use the notation $f(\cdot) \asymp g(\cdot)$ to indicate that $C<f(\cdot) / g(\cdot)<C^{\prime}$ for some $C<C^{\prime}$, on a given set $X$. The set $X$, in which this relation holds will be always mentioned; except in the case of sequences, when the relation will be naturally assumed to hold for all $j \in \mathbf{N}_{+}$.

Furthermore, we will use abbreviated notation $\zeta:=(\nu, \tau)$ for the boundary distance coordinates; in order to make some lengthy formulas more concise. Some other notations will be introduced later, when needed.

3.2. Local change of coordinates. We denote by $\alpha$ our unit speed parametrisation $^{1}$

$$
\tau \mapsto y(\tau) \in \gamma
$$

of the contour $\gamma$. For the sake of convenience we extend its domain of definition to the whole $\mathbf{R}$ as a periodic map. We assume that the parametrisation $\alpha$ is positively oriented, $(0,0) \in \gamma$ and $\alpha(0)=(0,0)$; this can be done without loss of generality.

We now begin to study the whole boundary distance coordinate parametrisation

$$
\zeta \mapsto y(\zeta) \in \mathcal{U}_{\delta}
$$

of the $\delta$-neighbourhood $\mathcal{U}_{\delta}$ of $\gamma$. We will denote this map in the sequel by $\varphi$.

Since $\alpha$ is unit speed parametrisation of $\gamma$, the interior unit normal vector of $\partial \Lambda$ at the point $y=\alpha(\tau)$ is given as

$$
\mathbf{e}_{i n}(y)=\left(-\alpha_{2}^{\prime}(\tau), \alpha_{1}^{\prime}(\tau)\right)
$$

because this vector is perpendicular to $\alpha^{\prime}(\tau)$, points into interior domain due to our choice of positive orientation, and has unit norm. Hence the map $\varphi$ is given by the formula

$$
\varphi(\zeta)=\left(\varphi_{1}(\zeta), \varphi_{2}(\zeta)\right)=\left(\alpha_{1}(\tau)-\nu \alpha_{2}^{\prime}(\tau), \alpha_{2}(\tau)+\nu \alpha_{1}^{\prime}(\tau)\right) .
$$

One can also show that if $\delta$ is small enough, which is assumed to be the case, then any point $y \in \mathcal{U}_{\delta}$ has unique representation

$$
y=y^{\prime}+\nu \mathbf{e}_{i n}\left(y^{\prime}\right),
$$

where $y^{\prime} \in \gamma$ and $\nu$ is the distance of $y$ to $\gamma$.

For the derivatives of $\varphi$ we obtain

$$
\begin{aligned}
\left|\partial_{\tau} \varphi(\zeta)\right| & =\left(\left(\alpha_{1}^{\prime}(\tau)-\nu \alpha_{2}^{\prime \prime}(\tau)\right)^{2}+\left(\alpha_{2}^{\prime}(\tau)+\nu \alpha_{1}^{\prime \prime}(\tau)\right)^{2}\right)^{1 / 2} \\
& =\left(1+2\left(\alpha_{2}^{\prime}(\tau) \alpha_{1}^{\prime \prime}(\tau) \alpha_{1}^{\prime}(\tau) \alpha_{2}^{\prime \prime}(\tau)\right) \nu+\left|\alpha^{\prime \prime}(\tau)\right|^{2} \nu^{2}\right)^{1 / 2}, \\
\left|\partial_{\nu} \varphi(\zeta)\right| & =\left(\left(-\alpha_{2}^{\prime}(\tau)\right)^{2}+\left(\alpha_{1}^{\prime}(\tau)\right)^{2}\right)^{1 / 2} \equiv 1,
\end{aligned}
$$

since $\alpha$ has unit speed. These identities will be used later.

We now begin to estimate the Jacobian of the map $\varphi$, denoted by $\left|J_{\varphi}\right|$ in the sequel. We have

$$
\begin{array}{ll}
\partial_{\tau} \varphi_{1}(\zeta)=\alpha_{1}^{\prime}(\tau)-\nu \alpha_{2}^{\prime \prime}(\tau), & \partial_{\nu} \varphi_{1}(\zeta)=-\alpha_{2}^{\prime}(\tau) \\
\partial_{\tau} \varphi_{2}(\zeta)=\alpha_{2}^{\prime}(\tau)+\nu \alpha_{1}^{\prime \prime}(\tau), & \partial_{\nu} \varphi_{2}(\zeta)=+\alpha_{1}^{\prime}(\tau) .
\end{array}
$$

\footnotetext{
${ }^{1}$ Curve length parametrisation of a simple $C^{1}$-curve is always unit speed parametrisation of that curve.
} 
Hence

$$
\left|J_{\varphi}\right|=\left(\alpha_{1}^{\prime}(\tau)-\nu \alpha_{2}^{\prime \prime}(\tau)\right) \alpha_{1}^{\prime}(\tau)-\left(-\alpha_{2}^{\prime}(\tau)\right)\left(\alpha_{2}^{\prime}(\tau)+\nu \alpha_{1}^{\prime \prime}(\tau)\right)
$$

and since $\left(\alpha_{1}^{\prime}(\tau)\right)^{2}+\left(\alpha_{2}^{\prime}(\tau)\right)^{2} \equiv 1$, we have

$$
\left|J_{\varphi}\right|=1+\nu\left(\alpha_{2}^{\prime}(\tau) \alpha_{1}^{\prime}(\tau)-\alpha_{1}^{\prime}(\tau) \alpha_{2}^{\prime}(\tau)\right)=: 1+\omega(\zeta) .
$$

Thus $\left|J_{\varphi}\right| \asymp 1$ on the set $\left\{\zeta \in \mathbf{R}^{2}:|\nu|<\varepsilon, \tau \in \mathbf{R}\right\}$ if

$$
\varepsilon<\left(\sup _{\tau \in \mathbf{R}}\left|\alpha_{2}^{\prime}(\tau) \alpha_{1}^{\prime}(\tau)-\alpha_{1}^{\prime}(\tau) \alpha_{2}^{\prime}(\tau)\right|\right)^{-1} .
$$

Hence one can verify that $\varphi$ defines a $C^{2}$-diffeomorphism

$$
\varphi: Q_{\varepsilon} \rightarrow \varphi\left(Q_{\varepsilon}\right),
$$

where $Q_{\varepsilon}=\left\{\zeta \in \mathbf{R}^{2}:|\nu|<\varepsilon,|\tau|<\varepsilon\right\}$, by using the inverse function theorem; if $\varepsilon>0$ is small enough, which is also needed for $\varphi$ to to be injective. (See appendix on [4] for similar construction with more details).

3.3. Construction of the sequence. In the sequel we denote

$$
h(\zeta):=\nu^{m} g(\nu, \tau)
$$

where $g$ is as in (1.1) in the definition of the domain $\Omega$. We will also use the notation

$$
\mathbf{h}(y):=h\left(\varphi^{-1}(y)\right)
$$

for the same function expressed in the $y$-coordinate system.

We will assume that the diffeomorphism (3.4) exists for some $\varepsilon>e^{-1}$; this can be done without loss of generality, and we will come back to this assumption when we have defined the sequence $\left(\Psi_{j}\right)_{j=1}^{\infty}$.

We now define the following sets in the $(\zeta, z)$-coordinate space

$$
\begin{aligned}
& V=\left\{\zeta \in \mathbf{R}^{2}: 0<\nu<e^{-1},|\tau| \leq \kappa \nu^{1 / 2}\right\}, \\
& \mathbf{V}=\left\{(\zeta, z) \in \mathbf{R}^{3}: \zeta \in V,-h(\zeta)<z<0\right\} ;
\end{aligned}
$$

and the following sets in the $x$-coordinate space

$$
\mathcal{V}=\varphi(V), \quad \mathfrak{V}=\left\{(y, z) \in \mathbf{R}^{3}:-\mathbf{h}(y) \leq z \leq 0, y \in \mathcal{V}\right\}
$$

Let $F:=\varphi \times i d$ be the map $(\zeta, z) \mapsto(\varphi(\zeta), z)$. We denote

$$
\Phi:=\Psi \circ F \quad \text { if } \Psi \in C_{c}^{\infty}(\overline{\mathfrak{V}} \backslash \overline{0}),
$$

where $\overline{0}:=(0,0,0)$. Same notation will be also used in the following way

$$
\Phi:=\Psi \circ \varphi \quad \text { if } \Psi \in C_{c}^{\infty}(\overline{\mathcal{V}} \backslash \overline{0}),
$$

where $\overline{0}:=(0,0)$.

We now define space $\mathcal{H}(\mathfrak{V} ; \mathcal{V})$ to be completion of the space $C_{c}^{\infty}(\overline{\mathfrak{V}} \backslash \overline{0})$, with respect to similar norm as in $\mathcal{H}(\Omega ; \Lambda)$; but $\Omega$ and $\Lambda$ replaced by $\mathfrak{V}$ and $\mathcal{V}$. We also define space $\mathcal{H}(\mathbf{V} ; V)$ to be similar completion of the space $C_{c}^{\infty}(\overline{\mathbf{V}} \backslash \overline{0})$; with the obvious exception been that in the definition of the norm all derivatives and integrals are taken over $(\zeta, z)$-coordinates. 
Since $\left|J_{\varphi}\right| \asymp 1$ on the set $\mathcal{V}$, it holds that $\left|J_{F}\right| \asymp 1$ on the set $\mathfrak{V}$, and one can show that the map $\Psi \mapsto \Phi$, from $C_{c}^{\infty}(\overline{\mathfrak{V}} \backslash \overline{0})$ to $C_{c}^{\infty}(\overline{\mathbf{V}} \backslash \overline{0})$, can be extended to an isomorphism

$$
L_{F}: \mathcal{H}(\mathfrak{V} ; \mathcal{V}) \rightarrow \mathcal{H}(\mathbf{V} ; V), \quad \Psi \mapsto \Phi ;
$$

where $\Phi=\Psi \circ F$ is defined almost everywhere in $\mathbf{V}$ (see Theorem 3.41 in [1] for details). ${ }^{2}$ Inverse operator $L_{F}^{-1}=L_{F^{-1}}$ is defined in the obvious way. Existence of the isomorphism $L_{F}$ is equivalent to the fact that

$$
\|\Psi ; \mathcal{H}(\mathfrak{V} ; \mathcal{V})\| \asymp\|\Phi ; \mathcal{H}(\mathbf{V} ; V)\|
$$

on the whole space $\mathcal{H}(\mathfrak{V} ; \mathcal{V})$. Similarly, the map $\Psi \mapsto \Phi$, from $C_{c}^{\infty}(\overline{\mathcal{V}} \backslash \overline{0})$ to $C_{c}^{\infty}(\bar{V} \backslash \overline{0})$, can be extended to an isomorphism

$$
L_{\varphi}: L^{2}(\mathcal{V}) \rightarrow L^{2}(V), \quad \Psi \mapsto \Phi
$$

where $\Phi=\Psi \circ \varphi$ is defined almost everywhere in $V$. The existence of this isomorphism is equivalent to similar relation as in (3.7) between the $L^{2}$-norms on $\mathcal{V}$ and $V$.

In the sequel we will denote

$$
\Phi:=\left.L_{F} \Psi\right|_{\mathfrak{V}} \quad \text { for any } \Psi \in \mathcal{H}(\Omega ; \Lambda)
$$

where $\left.\Psi\right|_{\mathfrak{V}} \in \mathcal{H}(\mathfrak{V} ; \mathcal{V})$ is the restriction of $\Psi$ to domain $\mathfrak{V}$, defined almost everywhere. And again, the same notation will be used also in the following sense

$$
\Phi:=\left.L_{\varphi} \Psi\right|_{\mathcal{V}} \quad \text { for any } \Psi \in L^{2}(\Lambda)
$$

where $\left.\Psi\right|_{\mathcal{V}} \in L^{2}(\Lambda)$ is the restriction of $\Psi$ to domain $\mathcal{V}$, defined almost everywhere. The later notation will be used for traces $\Psi(\cdot, 0)$ of functions $\Psi \in \mathcal{H}(\Omega ; \Lambda)$; which justifies the use of this notation in two seemingly different ways.

Next we will fix the sequence $\left(\Phi_{j}\right)_{j=1}^{\infty}$, which will give us the singular sequence $\left(\Psi_{j}\right)_{j=1}^{\infty}$ via the transformation $L_{F}^{-1}$.

For this purpose, we will seek for an approximate solution to the differential equation

$$
-\partial_{\nu}\left(g_{0} \nu^{m} \partial_{\nu} f(\nu)\right)=\lambda f(\nu)
$$

on the interval $(0, \infty]$; where

$$
g_{0}:=g(0,0)
$$

and $g$ is as in (3.5), this abbreviation will be used in the sequel.

Let

$$
U(\nu):=\exp \left(\frac{i \sqrt{\lambda g_{0}^{-1}} \nu^{-m / 2+1}}{-m / 2+1}\right)
$$

then

$$
U^{\prime}(\nu)=i \sqrt{\lambda g_{0}^{-1}} \nu^{-m / 2} \exp \left(\frac{i \sqrt{\lambda g_{0}^{-1}} \nu^{-m / 2+1}}{-m / 2+1}\right)=i \sqrt{\lambda g_{0}^{-1}} \nu^{-m / 2} U(\nu)
$$

\footnotetext{
${ }^{2}$ Theorem 3.41 in [1] considers only Sobolev spaces, but can be easily modified to our case.
} 
and

$$
\begin{aligned}
U^{\prime \prime}(\nu) & =\left(-i \frac{m}{2} \sqrt{\lambda g_{0}^{-1}} \nu^{-m / 2-1}-\lambda g_{0}^{-1} \nu^{-m}\right) \exp \left(\frac{i \sqrt{\lambda g_{0}^{-1}} \nu^{-m / 2+1}}{-m / 2+1}\right) \\
& =\left(-i \frac{m}{2} \sqrt{\lambda g_{0}^{-1}} \nu^{-m / 2-1}-\lambda g_{0}^{-1} \nu^{-m}\right) U(\nu)
\end{aligned}
$$

In particular,

$$
-\partial_{\nu}\left(g_{0} \nu^{m} \partial_{\nu} U(\nu)\right)=\lambda U(\nu)+i \frac{m-2}{2} \sqrt{\lambda g_{0}^{-1}} g_{0} \nu^{m / 2-1} U(\nu)
$$

where

$$
i \frac{m-2}{2} \sqrt{\lambda g_{0}^{-1}} g_{0} \nu^{m / 2-1} U(\nu) \rightarrow 0, \quad \text { as } \nu \rightarrow 0,
$$

since $m>2$. Thus $U$ is a approximate solution of $(3.9)$ on the interval $(0, \infty]$, in vicinity of the point $\nu=0$.

We are now ready to start constructing our sequence. We set

$$
\Phi_{j}(\zeta, z):=\Phi_{j}(\zeta):=a_{j} U(\nu) X_{j}(\zeta),
$$

where $a_{j}$ is a normalization constant to be fixed later and

$$
X_{j}(\zeta)=\chi\left(-\ln \nu-2^{j}\right) \chi\left(2^{j+1}+\ln \nu\right) \chi\left(\ln \kappa-2^{j}+\ln |\tau|\right),
$$

whit $\chi$ been any smooth non-negative cut-off function, such that

$$
\chi(t)= \begin{cases}1, & \text { for } t \geq 1 \\ 0, & \text { for } t \leq 0\end{cases}
$$

In particular $X_{j}$ is chosen to satisfy, for $k=1,2$,

$$
\left|\partial_{\nu}^{k} X_{j}(\zeta)\right| \leq C \nu^{-k} \quad \text { and } \quad\left|\partial_{\tau}^{k} X_{j}(\zeta)\right| \leq C|\tau|^{-k}
$$

With this definition $\operatorname{supp}\left(\Phi_{j}\right)$ is included in the domain $\mathfrak{B}_{j}$ with the base $B_{j}$, where

$$
\begin{aligned}
\mathfrak{B}_{j} & =\left\{(\zeta, z) \in \mathbf{R}^{3}:-h(\zeta) \leq z \leq 0, \zeta \in B_{j}\right\}, \\
B_{j} & =\left\{\zeta \in \mathbf{R}^{2}: e^{-2^{j+1}} \leq \nu \leq e^{-2^{j}},-\kappa e^{-2^{j}} \leq \tau \leq+\kappa e^{-2^{j}}\right\} .
\end{aligned}
$$

We also denote

$$
\begin{aligned}
& C_{j}=\left\{\zeta \in \mathbf{R}^{2}: e^{-2^{j+1}+1} \leq \nu \leq e^{-2^{j}-1},-\kappa e^{-2^{j}} \leq \tau \leq+\kappa e^{-2^{j}}\right\}, \\
& D_{j}=\left\{\zeta \in \mathbf{R}^{2}: e^{-2^{j+1}} \leq \nu \leq e^{-2^{j}},-\kappa e^{-2^{j}-1} \leq \tau \leq+\kappa e^{-2^{j}-1}\right\} .
\end{aligned}
$$

Notice that then

$$
\operatorname{mes}_{2} B_{j} \asymp \exp \left(-2^{j+1}\right) .
$$

and

$$
\operatorname{mes}_{2}\left(C_{j} \cap D_{j}\right) \asymp \exp \left(-2^{j+1}\right) .
$$

The sets $B_{j}, C_{j}$ and $D_{j}$ are contained in the set $V$. By Taylor expanding the function $g$ in the definition on $h$ and using the relation $|\tau| \leq \kappa \nu^{1 / 2}$ in the definition of the set $V$, we get

$$
\left|h(\zeta)-g_{0} \nu^{m}\right| \leq C \nu^{m+1 / 2} \text { on the set } V .
$$


We can now define functions

$$
\Psi_{j}(x):=\Psi_{j}(y):=\Phi_{j}\left(\varphi^{-1}(y)\right) .
$$

Which are the functions $\Phi_{j}$ expressed in the $(y, z)$-coordinate system. Clearly

$$
\operatorname{supp}\left(\Psi_{j}\right) \subset \varphi\left(B_{j}\right) \subset \mathcal{V} \text { for all } j
$$

(or $\operatorname{supp}\left(\Psi_{j}\right) \subset \mathfrak{V}$, if considered as a function of $x$ ). A similar structure of a singular sequence was used in [12] for the Steklov problem in peak-shaped domains.

We now come back to our assumption that the diffeomorphism (3.4) exists for some $\varepsilon>e^{-1}$. If this would not be the case, then any $\varepsilon>0$ for which the diffeomorphism (3.4) exists, satisfies the inequality $\varepsilon>e^{-2^{n}}$ for some $n \in \mathbf{N}, n>1$. In this case, we would choose $e^{-2^{n}}$ instead of $e^{-1}$ in the definition (3.6) of the set $V$, and define functions $\Phi_{j}$ as before, but only for values $j \geq n$. After this we would define $\widetilde{\Psi}_{j}(x):=\widetilde{\Psi}_{j}(y):=\Phi_{j+n-1}\left(\varphi^{-1}(y)\right)$ for all $j \in \mathbf{N}_{+}$. The proof that $\left(\widetilde{\Psi}_{j}\right)_{j=1}^{\infty}$ is a singular sequence, would be almost the same as the proof we are about to begin, we would just have to take into account the change of indexing at every step. For this reason we can assume without loss generality that the diffeomorphism (3.4) exists for some $\varepsilon>e^{-1}$.

In order to show that $\left(\Psi_{j}\right)_{j=1}^{\infty}$ is a singular sequence, we need to estimate the norms $\left\|\Psi_{j} ; \mathcal{H}(\Omega ; \Lambda)\right\|$. This will be more conveniently done in the $(\zeta, z)$-coordinates by using functions $\Phi_{j}$.

Due to (3.1) and (3.2) the gradient vector $\nabla_{x} \Psi(x)$ is represented in the $(\zeta, z)$ coordinates as

$$
\nabla_{x} \Psi(x)=\nabla_{x} \Psi(\varphi(\zeta), z)=\frac{1}{\left|\partial_{\tau} \varphi(\zeta)\right|} \partial_{\tau} \Phi(\zeta, z) \mathbf{e}_{\tau}+\partial_{\nu} \Phi(\zeta, z) \mathbf{e}_{\nu}+\partial_{z} \Phi(\zeta, z) \mathbf{e}_{z}
$$

since the unit vectors

$$
\mathbf{e}_{\tau}:=\frac{\partial_{\tau} \varphi(\zeta)}{\left|\partial_{\tau} \varphi(\zeta)\right|}, \quad \mathbf{e}_{\nu}:=\frac{\partial_{\nu} \varphi(\zeta)}{\left|\partial_{\nu} \varphi(\zeta)\right|} \quad \text { and } \quad \mathbf{e}_{z}
$$

form a orthonormal basis at the point $x=(\varphi(\zeta), z)$ (see chapter 7 in [18]); hence for any two functions $\Psi, \tilde{\Psi} \in \mathcal{H}(\Omega, \Lambda)$ with local representations $\Phi, \tilde{\Phi}$ in the $(\zeta, z)$ coordinate system, the relation

$$
\begin{aligned}
\left(\nabla_{x} \Psi(x), \nabla_{x} \tilde{\Psi}(x)\right) & =\left(\nabla_{x} \Psi(\varphi(\zeta), x), \nabla_{x} \tilde{\Psi}(\varphi(\zeta), z)\right) \\
& =\frac{\partial_{\tau} \Phi(\zeta, z) \partial_{\tau} \tilde{\Phi}(\zeta, z)}{\left|\partial_{\tau} \varphi(\zeta)\right|^{2}}+\partial_{\nu} \Phi(\zeta, z) \partial_{\nu} \tilde{\Phi}(\zeta, z)+\partial_{z} \Phi(\zeta, z) \partial_{z} \tilde{\Phi}(\zeta, z)
\end{aligned}
$$

holds for almost every $x \in \Omega$ with $y \in Q_{+}$. Thus, if $\Psi(x)=\Psi(y)$ and $\tilde{\Psi}(x)=\tilde{\Psi}(y)$, then

$$
\left(\nabla_{x} \Psi, \nabla_{x} \tilde{\Psi}\right)_{\Omega}=\int_{V} h(\nu)\left(\frac{\partial_{\tau} \Phi(\zeta) \partial_{\tau} \tilde{\Phi}(\zeta)}{\left|\partial_{\tau} \varphi(\zeta)\right|^{2}}+\partial_{\nu} \Phi(\zeta) \partial_{\nu} \tilde{\Phi}(\zeta)\right) d \zeta
$$

if either $\operatorname{supp}(\Psi) \subset \mathcal{V}$ or $\operatorname{supp}(\tilde{\Psi}) \subset \mathcal{V}$.

Now

$$
\begin{aligned}
\frac{1}{\left|\partial_{\tau} \varphi(\zeta)\right|^{2}} & =1-\frac{2\left(\alpha_{2}^{\prime}(\tau) \alpha_{1}^{\prime \prime}(\tau)-\alpha_{1}^{\prime}(\tau) \alpha_{2}^{\prime \prime}(\tau)\right) \nu+\left|\alpha^{\prime \prime}(\tau)\right|^{2} \nu^{2}}{1+2\left(\alpha_{2}^{\prime}(\tau) \alpha_{1}^{\prime \prime}(\tau)-\alpha_{1}^{\prime}(\tau) \alpha_{2}^{\prime \prime}(\tau)\right) \nu+\left|\alpha^{\prime \prime}(\tau)\right|^{2} \nu^{2}} \\
& =: 1-G(\zeta) .
\end{aligned}
$$


Hence, due to definition (3.16)

$$
|G(\zeta)| \leq \frac{C \nu}{\left|\partial_{\tau} \varphi(\zeta)\right|^{2}} \leq \frac{C \exp \left(-2^{j}\right)}{\left|\partial_{\tau} \varphi(\zeta)\right|^{2}}
$$

on the sets $B_{j}$.

We calculate some estimates for the functions (3.14). Since $|U(\nu)| \equiv 1,\left|X_{j}(\zeta)\right| \leq$ 1 and $\left|J_{\varphi}\right| \asymp 1$ on the set $V$, we get

$$
\left\|\Psi_{j} ; L^{2}(\Lambda)\right\|^{2} \leq a_{j}^{2} \int_{B_{j}}\left|J_{\varphi}\right| d \zeta \leq C a_{j}^{2} \operatorname{mes}_{2} B_{j} \leq C a_{j}^{2} \exp \left(-2^{j+1}\right),
$$

and since $X_{j}(\zeta)=1$ for all $\zeta \in C_{j} \cap D_{j}$, similar calculation shows that

$$
\left\|\Psi_{j} ; L^{2}(\Lambda)\right\|^{2} \geq a_{j}^{2} \int_{B_{j} \cap C_{j}}\left|J_{\varphi}\right| d \zeta \geq C a_{j}^{2} \exp \left(-2^{j+1}\right) .
$$

Moreover, using (3.15), (3.19) and $h(\nu) \leq C \nu^{m}$ on $V$, we get

$$
\begin{aligned}
\left\|\nabla_{x} \Psi_{j} ; L^{2}(\Omega)\right\|^{2} & \leq C a_{j}^{2} \int_{B_{j}} \nu^{m}\left(\left|\partial_{\nu} X_{j}(\zeta)\right|^{2}+\left|\partial_{\tau} X_{j}(\zeta)\right|^{2}+\nu^{-m}\right)\left|J_{\varphi}\right| d \zeta \\
& \leq C^{\prime} a_{j}^{2}\left(\int_{B_{j}}\left(\nu^{m-2}+1\right) d \zeta+\int_{B_{j} \backslash D_{j}}|\tau|^{-2} \nu^{m} d \zeta\right)
\end{aligned}
$$

since $\nabla_{x} \Psi_{j}=\nabla_{y} \Psi_{j}, \operatorname{supp}\left(\nabla_{y} \Psi_{j}\right) \subset \varphi\left(B_{j}\right) \subset \mathcal{V}, \operatorname{supp}\left(\partial_{\tau} X_{j}\right) \subset B_{j} \backslash D_{j}$ and

$$
\frac{1}{\left|\partial_{\tau} \varphi(\zeta)\right|^{2}} \leq C \quad \text { on the set } V \text {. }
$$

Thus, due to (3.17)

$$
\begin{aligned}
& \left\|\nabla_{x} \Psi_{j} ; L^{2}(\Omega)\right\|^{2} \leq C a_{j}^{2}\left(\operatorname{mes}_{2} B_{j}+2 \int_{+\kappa \exp \left(-2^{j}-1\right)}^{+\kappa \exp \left(-2^{j}\right)} \tau^{-2} d \tau \int_{\exp \left(-2^{j+1}\right)}^{\exp \left(-2^{j}\right)} \nu^{m} d \nu\right) \\
& \leq C a_{j}^{2}\left(\exp \left(-2^{j+1}\right)+\exp \left(+2^{j}\right) \exp \left(-(m+1) 2^{j}\right)\right) \leq C^{\prime} a_{j}^{2} \exp \left(-2^{j+1}\right)
\end{aligned}
$$

since $m>2$. Hence, putting

$$
a_{j}:=\exp \left(+2^{j}\right)
$$

we have

$$
\left\|\Psi_{j} ; \mathcal{H}(\Omega ; \Lambda)\right\| \asymp 1 .
$$

Thus the sequence $\left(\Psi_{j}\right)_{j=1}^{\infty}$ satisfies the conditions i) and iii) in the Definition 2.1, since $K_{\Omega}$ is defined on the whole $\mathcal{H}(\Omega ; \Lambda)$. Moreover, since $\operatorname{supp}\left(\Psi_{j}\right) \cap \operatorname{supp}\left(\Psi_{k}\right)=\varnothing$ for $j \neq k$, the sequence $\left(\Psi_{j}\right)_{j=1}^{\infty}$ is orthogonal. Hence

$$
\Psi_{j} \stackrel{w}{\rightarrow} 0 \quad \text { as } \quad j \rightarrow \infty,
$$

due to the Lemma 3.23 below, so the condition ii) in the Definition 2.1 is also satisfied. 
3.23. Lemma. If $\left(\Psi_{j}\right)_{j=1}^{\infty}$ is an orthogonal sequence in a Hilbert space $\mathcal{H}$ and

$$
\left\|\Psi_{j} ; \mathcal{H}\right\| \asymp 1
$$

then $\Psi_{j} \stackrel{w}{\rightarrow} 0$ as $j \rightarrow \infty$.

Proof. Let $\Upsilon_{j}:=\left\|\Psi_{j} ; \mathcal{H}\right\|^{-1} \Psi_{j}$, then the sequence $\left(\Upsilon_{j}\right)_{j=1}^{\infty}$ is orthonormal and can be extended to a orthonormal base $\left(\mathbf{e}_{k}\right)_{k=1}^{\infty}$ of $\mathcal{H}$; where $\mathbf{e}_{k_{j}}=\Upsilon_{j}$ for some subsequence $\left(k_{j}\right)_{j=1}^{\infty} \subset \mathbf{N}_{+}$. Since every $\Psi \in \mathcal{H}$ has a representation

$$
\Psi=\sum_{k=1}^{\infty}\left(\Psi, \mathbf{e}_{k}\right) \mathbf{e}_{k}
$$

(where $(\cdot, \cdot)$ is the inner product of $\mathcal{H}$ ) it must be true that

$$
\left(\Psi, \mathbf{e}_{k_{j}}\right)=\left(\Psi, \Upsilon_{j}\right) \rightarrow 0 \quad \text { as } \quad j \rightarrow \infty .
$$

The claim now follows from (3.24) and the definition of functions $\Upsilon_{j}$.

To conclude that $\left(\Psi_{j}\right)_{j=1}^{\infty}$ is a singular sequence at the given point $\mu=(1+\lambda)^{-1} \in$ $(0,1)$, we still need to show that also the condition $i v)$ in the Definition 2.1 holds for $\left(\Psi_{j}\right)_{j=1}^{\infty}$. It will be done by verifying that the norm

$$
\left\|K_{\Omega} \Psi_{j}-(1+\lambda)^{-1} \Psi_{j} ; \mathcal{H}(\Omega ; \Lambda)\right\|=\sup _{\Psi \in \mathbf{S}} \mid\left\langle\left(K_{\Omega}-(1+\lambda)^{-1} \Psi_{j}, \Psi\right\rangle_{\Omega}\right|,
$$

where $\mathbf{S}$ denotes the unit sphere in $\mathcal{H}(\Omega ; \Lambda)$, tends to zero as $j \rightarrow \infty$. For this purpose we will estimate the expression

$$
\mid\left\langle\left(K_{\Omega}-(1+\lambda)^{-1} \Psi_{j}, \Psi\right\rangle_{\Omega}\right|
$$

from above, for arbitrary $\Psi$ with $\|\Psi ; \mathcal{H}(\Omega ; \Lambda)\|=1$.

We set

$$
\bar{\Psi}(y):=\mathbf{h}(y)^{-1} \int_{-\mathbf{h}(y)}^{0} \Psi(y, z) d z \text { and } \bar{\Phi}(\zeta):=h(\zeta)^{-1} \int_{-h(\zeta)}^{0} \Phi(\zeta, z) d z .
$$

Clearly, if $\Psi \in L^{2}(\mathcal{V})$ and $\Phi$ is as in (3.8), then the equalities

$$
\bar{\Phi}(\zeta)=\bar{\Psi}(\varphi(\zeta)) \text { and } \bar{\Psi}(y)=\bar{\Phi}\left(\varphi^{-1}(y)\right)
$$

are valid for almost every $\zeta \in V$ and almost every $y \in \mathcal{V}$, respectively.

Since the functions $\Psi_{j}$ do not depend on $z$, we have

$$
\begin{aligned}
& \left(\nabla_{x} \Psi_{j}, \nabla_{x} \Psi\right)_{\Omega}-\lambda\left(\Psi_{j}, \Psi(\cdot, 0)\right)_{\Lambda}=\left(\mathbf{h} \nabla_{y} \Psi_{j}, \overline{\nabla_{y} \Psi}\right)_{\mathcal{V}}-\lambda\left(\Psi_{j}, \Psi(\cdot, 0)\right)_{\mathcal{V}} \\
& =\left(\left(\mathbf{h}-g_{0} \nu^{m}\right) \nabla_{y} \Psi_{j}, \nabla_{y} \bar{\Psi}\right)_{\mathcal{V}}+\left(\left(g_{0} \nu^{m} \nabla_{y} \Psi_{j}, \nabla_{y} \bar{\Psi}\right)_{\mathcal{V}}-\lambda\left(\Psi_{j}, \bar{\Psi}\right)_{\mathcal{V}}\right) \\
& \quad+\left(\mathbf{h} \nabla_{y} \Psi_{j}, \overline{\nabla_{y} \Psi}-\nabla_{y} \bar{\Psi}\right)_{\mathcal{V}}-\lambda\left(\Psi_{j}, \Psi(\cdot, 0)-\bar{\Psi}\right)_{\mathcal{V}} \\
& =: I_{0}+I_{1}+I_{2}-\lambda I_{3} .
\end{aligned}
$$

Next we will conclude that $I_{k} \rightarrow 0$ as $j \rightarrow \infty$, for any $\Psi$ with $\|\Psi ; \mathcal{H}(\Omega ; \Lambda)\|=1$, and for all $k=0,1,2,3$; which finishes the proof.

3.4. Estimate for $\boldsymbol{I}_{\mathbf{3}}$. In our estimation of the term $I_{3}$ we will need the Lemma 3.25 below. 
3.25. Lemma. The following inequalities hold true:

$$
\begin{aligned}
& \left\|\mathbf{h}^{-1 / 2}(\Psi(\cdot, 0)-\bar{\Psi}) ; L^{2}(\mathcal{V})\right\| \leq C\left\|\partial_{z} \Psi ; L^{2}(\mathfrak{V})\right\|, \\
& \left\|\mathbf{h}^{-1 / 2}(\Psi(\cdot,-\mathbf{h}(\cdot))-\bar{\Psi}) ; L^{2}(\mathcal{V})\right\| \leq C\left\|\partial_{z} \Psi ; L^{2}(\mathfrak{V})\right\| ;
\end{aligned}
$$

where $\Psi(\cdot, 0)$ is the trace of $\Psi$ on the surface $\Lambda$ and $\Psi(\cdot,-h(\cdot))$ is the trace of $\Psi$ on the surface $\Sigma \cap \overline{\mathfrak{V}}$.

Proof. Integrating by parts yields

$$
\mathbf{h}(y)^{-1} \int_{-\mathbf{h}(y)}^{0}(\mathbf{h}(y)+z) \partial_{z} \Psi(y, z) d z=\Psi(y, 0)-\bar{\Psi}(y) .
$$

Thus, by the Cauchy-Schwartz inequality

$$
|\Psi(y, 0)-\bar{\Psi}(y)|^{2} \leq \int_{-\mathbf{h}(y)}^{0}\left|\frac{\mathbf{h}(y)+z}{\mathbf{h}(y)}\right|^{2} d z \int_{-h(y)}^{0}\left|\partial_{z} \Psi(y, z)\right|^{2} d z \leq C \int_{-\mathbf{h}(y)}^{0}\left|\partial_{z} \Psi(y, z)\right|^{2} d z .
$$

Hence,

$$
\int_{\mathcal{V}}\left|\mathbf{h}(y)^{-1 / 2}(\Psi(y, 0)-\bar{\Psi}(y))\right|^{2} d y \leq C \int_{\mathcal{V}} \int_{-\mathbf{h}(y)}^{0}\left|\partial_{z} \Psi(y, z)\right|^{2} d z d y
$$

which is the inequality (3.26).

Similarly, integration by parts implies

$$
\bar{\Psi}(y)-\Psi(y,-\mathbf{h}(y))=\mathbf{h}(y)^{-1} \int_{-\mathbf{h}(y)}^{0} z \partial_{z} \Psi(y, z) d z
$$

and same type of estimation as above gives us also the inequality (3.27).

We have

$$
\left|I_{3}\right| \leq C\left\|\Psi_{j} ; L^{2}(\Lambda)\right\|\left\|\Psi(\cdot, 0)-\bar{\Psi} ; L^{2}(\Lambda)\right\| \leq C^{\prime}\left\|\mathbf{h}^{1 / 2} \mathbf{h}^{-1 / 2}(\Psi(\cdot, 0)-\bar{\Psi}) ; L^{2}(\Lambda)\right\|
$$
due to the Cauchy-Schwartz inequality, since

$$
\left\|\Psi_{j} ; \mathcal{H}(\Omega ; \Lambda)\right\| \asymp 1 \quad \text { and } \quad 1 \equiv \mathbf{h}(y)^{1 / 2} \mathbf{h}(y)^{-1 / 2}
$$

on the set $\Lambda$.

Now, by applying the Cauchy-Schwartz inequality again, and the Lemma 3.25 to the last expression in (3.28), we obtain

$$
\begin{aligned}
\left|I_{3}\right| & \leq\left\|\mathbf{h}^{1 / 2} ; L^{2}(\Lambda)\right\|\left\|\mathbf{h}^{-1 / 2}(\Psi(\cdot, 0)-\bar{\Psi}) ; L^{2}(\Lambda)\right\| \\
& \leq C \exp \left(-m 2^{j} / 2\right)\left\|\mathbf{h}^{-1 / 2}(\Psi(\cdot, 0)-\bar{\Psi}) ; L^{2}(\Lambda)\right\| \\
& \leq C^{\prime} \exp \left(-m 2^{j-1}\right)\left\|\partial_{z} \Psi ; L^{2}(\mathfrak{V})\right\| \leq C^{\prime} \exp \left(-m 2^{j-1}\right),
\end{aligned}
$$

since $\left\|\Psi_{j} ; L^{2}(\Lambda)\right\| \asymp 1$ and $\left\|\partial_{z} \Psi ; L^{2}(\mathfrak{V})\right\| \leq\|\Psi ; \mathcal{H}(\Omega ; \Lambda)\|=1$. Thus $I_{3} \rightarrow 0$ as $j \rightarrow \infty$.

3.5. Estimate for $\boldsymbol{I}_{\mathbf{2}}$. We begin our estimation for the term $I_{2}$ by stating and proving the Lemma 3.29 below. 
3.29. Lemma. The inequality

$$
\left\|\nu \mathbf{h}^{-1 / 2}\left(\overline{\nabla_{y} \Psi}-\nabla_{y} \bar{\Psi}\right) ; L^{2}(\mathcal{V})\right\| \leq C\left\|\partial_{z} \Psi ; L^{2}(\mathfrak{V})\right\|
$$

holds true for all $\Psi \in \mathcal{H}(\Omega, \Lambda)$.

Proof.

$$
\begin{aligned}
\nabla_{y} \bar{\Psi}(y)= & \nabla_{y}\left(\mathbf{h}(y)^{-1} \int_{-\mathbf{h}(y)}^{0} \Psi(y, z) d z\right)=-\frac{\nabla_{y} \mathbf{h}(y)}{\mathbf{h}(y)^{2}} \int_{-\mathbf{h}(y)}^{0} \Psi(y, z) d z \\
& +\mathbf{h}(y)^{-1}\left(\Psi(y,-\mathbf{h}(y)) \nabla_{y} \mathbf{h}(y)+\int_{-\mathbf{h}(y)}^{0} \nabla_{y} \Psi(y, z) d z\right) .
\end{aligned}
$$

Thus

$$
\overline{\nabla_{y} \Psi}-\nabla_{y} \bar{\Psi}=\mathbf{h}(y)^{-1} \nabla_{y} \mathbf{h}(y)(\bar{\Psi}(y)-\Psi(y,-\mathbf{h}(y))) .
$$

Now

$$
\nabla_{y} \mathbf{h}(y)=\nabla_{y} \mathbf{h}(\varphi(\zeta))=\frac{\partial_{\tau} h(\zeta)}{\left|\partial_{\tau} \varphi(\zeta)\right|} \mathbf{e}_{\tau}+\partial_{\nu} h(\zeta) \mathbf{e}_{\nu}
$$

and since $h(\zeta)=g(\zeta) \nu^{m}$, we get for all $\zeta \in V$,

$$
\left|\nabla_{y} \mathbf{h}(\varphi(\zeta))\right|=\left|\frac{\partial_{\tau} h(\zeta)}{\partial_{\tau} \varphi(\zeta)}\right|+\left|\left(\partial_{\nu} g(\zeta)\right) \nu^{m}+g(\zeta) m \nu^{m-1}\right| \leq C \nu^{m-1}
$$

Since $\mathbf{h} \asymp \nu^{m}$ on $\mathcal{V}$, we have

$$
\left|\mathbf{h}(y)^{-1} \nabla_{y} \mathbf{h}(y)\right| \leq C \nu^{-1}(y) \text { for all } y \in \mathcal{V} .
$$

Using (3.30), (3.31) we get

$$
\begin{aligned}
\left\|\nu \mathbf{h}^{-1 / 2}\left(\overline{\nabla_{y} \Psi}-\nabla_{y} \bar{\Psi}\right) ; L^{2}(\mathcal{V})\right\| & \leq C\left\|\mathbf{h}^{-1 / 2}(\bar{\Psi}-\Psi(\cdot,-\mathbf{h}(\cdot))) ; L^{2}(\mathcal{V})\right\| \\
& \leq C^{\prime}\left\|\partial_{z} \Psi ; L^{2}(\mathfrak{V})\right\|,
\end{aligned}
$$

where the last inequality is the inequality (3.27) from the Lemma 3.25 .

By use of the Lemma 3.29 and the equation

$$
\nu^{2}(y)=\mathbf{h}(y)\left|\nu(y) \mathbf{h}(y)^{-1 / 2}\right|^{2}
$$

we see that

$$
\begin{aligned}
\left|I_{2}\right| & \leq C\left\|\nu^{m-1} \nabla_{y} \Psi_{j} ; L^{2}(\mathcal{V})\right\|\left\|\nu \mathbf{h}^{-1 / 2}\left(\overline{\nabla_{y} \Psi}-\nabla_{y} \bar{\Psi}\right) ; L^{2}(\mathcal{V})\right\| \\
& \leq C^{\prime}\left\|\nu^{m-1} \nabla_{y} \Psi_{j} ; L^{2}(\mathcal{V})\right\|\left\|\partial_{z} \Psi ; L^{2}(\mathfrak{V})\right\| \\
& \leq C^{\prime \prime} \exp \left(-(m-2) 2^{j}\right)\|\Psi ; \mathcal{H}(\Omega ; \Lambda)\| \leq C^{\prime \prime} \exp \left(-(m-2) 2^{j}\right),
\end{aligned}
$$

since $\left\|\partial_{z} \Psi ; L^{2}(\mathfrak{V})\right\| \leq\|\Psi ; \mathcal{H}(\Omega ; \Lambda)\|=1$; using the estimate

$$
\nu^{2 m-2}(y) \leq C \exp \left(-2(m-2) 2^{j}\right) \text { for all } y \in \varphi\left(B_{j}\right),
$$


we get

$$
\begin{aligned}
\left\|\nu^{m-1} \nabla_{y} \Psi_{j} ; L^{2}(V)\right\| & =\left(\int_{\varphi\left(B_{j}\right)} \nu^{2 m-2}(y)\left|\nabla_{y} \Psi_{j}\right|^{2} d y\right)^{1 / 2} \\
& \leq C \exp \left(-(m-2) 2^{j}\right)\left(\int_{\varphi\left(B_{j}\right)} \nu^{m}(y)\left|\nabla_{y} \Psi_{j}\right|^{2} d y\right)^{1 / 2} \\
& \leq C^{\prime} \exp \left(-(m-2) 2^{j}\right)\left\|\nabla_{y} \Psi_{j} ; L^{2}(\Omega)\right\|
\end{aligned}
$$

Hence $I_{2} \rightarrow 0$ as $j \rightarrow \infty$.

3.6. Estimate for $\boldsymbol{I}_{\mathbf{1}}$. Due to (3.18) and (3.20)

$$
\begin{aligned}
\left(g_{0} \nu^{m} \nabla_{y} \Psi_{j}, \nabla_{y} \bar{\Psi}\right)_{\Lambda}= & \int_{B_{j}} g_{0} \nu^{m}\left(\partial_{\tau} \Phi_{j}(\zeta) \partial_{\tau} \bar{\Phi}(\zeta)+\partial_{\nu} \Phi_{j}(\zeta) \partial_{\nu} \bar{\Phi}(\zeta)\right)\left|J_{\varphi}\right| d \zeta \\
& -\int_{B_{j}} g_{0} \nu^{m} G(\zeta) \partial_{\tau} \Phi_{j}(\zeta) \partial_{\tau} \bar{\Phi}(\zeta)\left|J_{\varphi}\right| d \zeta=: I^{\prime}-I^{\prime \prime}
\end{aligned}
$$

We have

$$
\begin{aligned}
\left|I^{\prime \prime}\right| & \leq \int_{B_{j}} g_{0} \nu^{m}\left|G(\zeta) \partial_{\tau} \Phi_{j}(\zeta) \partial_{\tau} \bar{\Phi}(\zeta)\right|\left|J_{\varphi}\right| d \zeta \\
& \leq\left(\int_{B_{j}} g_{0} \nu^{m}|G(\zeta)|\left|\partial_{\tau} \Phi_{j}(\zeta)\right|^{2}|| J_{\varphi} \mid d \zeta\right)^{1 / 2}\left(\int_{B_{j}} g_{0} \nu^{m}|G(\zeta)|\left|\partial_{\tau} \bar{\Phi}(\zeta)\right|^{2}\left|J_{\varphi}\right| d \zeta\right)^{1 / 2}
\end{aligned}
$$

by using the Cauchy-Schwartz inequality with respect to weighted $L^{2}$-norm

$$
\left\|f ; L^{2}\left(B_{j} ; w_{1}\right)\right\|:=\left(\int_{B_{j}}|f(\zeta)|^{2} w_{1}(\zeta) d \zeta\right)^{1 / 2}, \quad w_{1}(\zeta):=G(\zeta) g_{0} \nu^{m}\left|J_{\varphi}\right| .
$$

From (3.21) we get

$$
\begin{aligned}
& \left(\int_{B_{j}} g_{0} \nu^{m}|G(\zeta)|\left|\partial_{\tau} \Phi(\zeta)\right|^{2}|| J_{\varphi} \mid d \zeta\right)^{1 / 2} \\
& \leq C \exp \left(-2^{j-1}\right)\left(\int_{B_{j}} \nu^{m} \frac{1}{\left|\partial_{\tau} \varphi(\zeta)\right|^{2}}\left|\partial_{\tau} \Phi(\zeta)\right|^{2}|| J_{\varphi} \mid d \zeta\right)^{1 / 2} .
\end{aligned}
$$

Hence, since

$$
\left\|\mathbf{h}^{1 / 2} \nabla_{y} \Psi ; L^{2}\left(\varphi\left(B_{j}\right)\right)\right\|=\left(\int_{B_{j}} h(\zeta)\left(\frac{1}{\left|\partial_{\tau} \varphi(\zeta)\right|^{2}}\left|\partial_{\tau} \Phi(\zeta)\right|^{2}+\left|\partial_{\nu} \Phi(\zeta)\right|^{2}\right)\left|J_{\varphi}\right| d \zeta\right)^{1 / 2}
$$

and $h \asymp \nu^{m}$ on $V$, we see that

$$
\left|I^{\prime \prime}\right| \leq C \exp \left(-2^{j}\right)\left\|\mathbf{h}^{1 / 2} \nabla_{y} \Psi_{j} ; L^{2}\left(\varphi\left(B_{j}\right)\right)\right\|\left\|\mathbf{h}^{1 / 2} \nabla_{y} \bar{\Psi} ; L^{2}\left(\varphi\left(B_{j}\right)\right)\right\| .
$$


Now

$$
\left\|\mathbf{h}^{1 / 2} \nabla_{y} \Psi_{j} ; L^{2}\left(\varphi\left(B_{j}\right)\right)\right\|=\left(\int_{\varphi\left(B_{j}\right)} \mathbf{h}(y)\left|\nabla_{y} \Psi_{j}(y)\right|^{2} d y\right)^{1 / 2}=\left\|\nabla_{x} \Psi_{j} ; L^{2}(\Omega)\right\|,
$$

since $\nabla_{x} \Psi_{j}=\nabla_{y} \Psi_{j}$ and $\operatorname{supp}\left(\nabla_{y} \Psi_{j}\right)=\varphi\left(B_{j}\right)$. And on the other hand

$$
\begin{aligned}
\left\|\mathbf{h}^{1 / 2} \nabla_{y} \bar{\Psi} ; L^{2}\left(\varphi\left(B_{j}\right)\right)\right\| & \leq\left\|\mathbf{h}^{1 / 2} \nabla_{y} \bar{\Psi} ; L^{2}\left(\varphi\left(B_{j}\right)\right)\right\| \\
& \leq\left\|\nabla_{x} \Psi ; L^{2}(\mathfrak{V})\right\| \leq\left\|\nabla_{x} \Psi ; L^{2}(\Omega)\right\|,
\end{aligned}
$$

due to the Lemma 3.32 below.

3.32. Lemma. The inequality

$$
\left\|\mathbf{h}^{1 / 2} \nabla_{y} \bar{\Psi} ; L^{2}(\mathcal{V})\right\| \leq C\left\|\nabla_{x} \Psi ; L^{2}(\mathfrak{V})\right\|
$$

is valid for all $\Psi \in \mathcal{H}(\Omega ; \Lambda)$.

Proof. If $\Psi \in \mathcal{H}(\Omega ; \Lambda)$, then

$$
\begin{aligned}
\left|\int_{-\mathbf{h}(\zeta)}^{0} \nabla_{y} \Psi(y, z) d z\right|^{2} & =\left|\int_{-\mathbf{h}(y)}^{0} \mathbf{h}(y)^{-1 / 2} \mathbf{h}(y)^{1 / 2} \nabla_{y} \Psi(y, z) d z\right|^{2} \\
& \leq \int_{-\mathbf{h}(y)}^{0} \mathbf{h}(y)^{-1} d z \int_{-\mathbf{h}(y)}^{0} \mathbf{h}(y)\left|\nabla_{y} \Psi(y, z)\right|^{2} d z \\
& =\mathbf{h}(y) \int_{-\mathbf{h}(y)}^{0}\left|\nabla_{y} \Psi(y, z)\right|^{2} d z
\end{aligned}
$$

by the Cauchy-Schwartz inequality. Applying this we get

$$
\int_{\mathcal{V}} \mathbf{h}(y)^{-1}\left|\int_{-\mathbf{h}(y)}^{0} \nabla_{y} \Psi(y, z) d z\right|^{2} d y \leq \int_{V} \int_{-\mathbf{h}(y)}^{0}\left|\nabla_{y} \Psi(y, z)\right|^{2} d y d z=\int_{\mathfrak{V}}\left|\nabla_{y} \Psi(y, z)\right|^{2} d x .
$$

In particular, we have

$$
\begin{aligned}
\left\|\mathbf{h}^{1 / 2} \overline{\nabla_{y} \Psi} ; L^{2}(\mathcal{V})\right\| & =\left(\int_{\mathcal{V}} \mathbf{h}(y)^{-1}\left|\int_{-h(y)}^{0} \nabla_{y} \Psi(y, z) d z\right|^{2} d y\right)^{1 / 2} \\
& \leq\left\|\nabla_{y} \Psi ; L^{2}(\mathfrak{V})\right\| .
\end{aligned}
$$

Since

$$
\mathbf{h}^{1 / 2}(y) \leq C \nu(y) \mathbf{h}^{-1 / 2}(y) \text { for all } y \in \mathcal{V}
$$

we have

$$
\left\|\mathbf{h}^{1 / 2} \nabla_{y} \bar{\Psi} ; L^{2}(\mathcal{V})\right\| \leq C\left\|\nu \mathbf{h}^{-1 / 2}\left(\nabla_{y} \bar{\Psi}-\overline{\nabla_{y} \Psi}\right) ; L^{2}(\mathcal{V})\right\|+\left\|\mathbf{h}^{1 / 2} \overline{\nabla_{y} \Psi} ; L^{2}(\mathcal{V})\right\|
$$

and the claim follows from the Lemma 3.29 and the inequality (3.33), since

$\left\|\nabla_{y} \Psi ; L^{2}(\mathfrak{V})\right\| \leq\left\|\nabla_{x} \Psi ; L^{2}(\mathfrak{V})\right\| \quad$ and $\quad\left\|\partial_{z} \Psi ; L^{2}(\mathfrak{V})\right\| \leq\left\|\nabla_{x} \Psi ; L^{2}(\mathfrak{V})\right\|$ for all $\Psi \in \mathcal{H}(\Omega ; \Lambda)$. 
Thus

$$
\left|I^{\prime \prime}\right| \leq C \exp \left(-2^{j}\right)\left\|\nabla_{x} \Psi_{j} ; L^{2}(\Omega)\right\|\left\|\nabla_{x} \Psi ; L^{2}(\Omega)\right\| \leq C^{\prime} \exp \left(-2^{j}\right),
$$

since $\left\|\Psi_{j} ; \mathcal{H}(\Omega ; \Lambda)\right\| \asymp 1$ and $\|\Psi ; \mathcal{H}(\Omega ; \Lambda)\|=1$. This shows that $I^{\prime \prime}$ tends to zero as $j \rightarrow \infty$.

Since $I_{1}=I^{\prime}+I^{\prime \prime}-\lambda\left(\Psi_{j}, \bar{\Psi}\right)_{\mathcal{V}}$, we still need to show that the term

$$
I^{\prime}-\lambda\left(\Psi_{j}, \bar{\Psi}\right)_{\Lambda}=: I^{\prime}-\lambda I^{-} \text {. }
$$

tends to zero as $j \rightarrow \infty$.

We have

$$
\begin{aligned}
I^{\prime}= & \int_{B_{j}} g_{0} \nu^{m}\left(\partial_{\tau} \Phi_{j}(\zeta) \partial_{\tau} \bar{\Phi}(\zeta)+\partial_{\nu} \Phi_{j}(\zeta) \partial_{\nu} \bar{\Phi}(\zeta)\right)\left|J_{\varphi}\right| d \zeta \\
= & \int_{B_{j}} g_{0} \nu^{m}\left(\partial_{\tau} \Phi_{j}(\zeta) \partial_{\tau} \bar{\Phi}(\zeta)+\partial_{\nu} \Phi_{j}(\zeta) \partial_{\nu} \bar{\Phi}(\zeta)\right) d \zeta \\
& +\int_{B_{j}} g_{0} \nu^{m}\left(\partial_{\tau} \Phi_{j}(\zeta) \partial_{\tau} \bar{\Phi}(\zeta)+\partial_{\nu} \Phi_{j}(\zeta) \partial_{\nu} \bar{\Phi}(\zeta)\right) \omega(\zeta) d \zeta=: I^{\circ}+I^{\circ \circ},
\end{aligned}
$$

since $\left|J_{\varphi}\right|=1+\omega(\zeta)$, as in (3.3).

Now

$$
\begin{aligned}
\left|I^{\circ \circ}\right| & \leq \int_{B_{j}}\left|g_{0} \nu^{m}\left(\partial_{\tau} \Phi_{j}(\zeta) \partial_{\tau} \bar{\Phi}(\zeta)+\partial_{\nu} \Phi_{j}(\zeta) \partial_{\nu} \bar{\Phi}(\zeta)\right)\right||\omega(\zeta)| d \zeta \\
& \leq C \exp \left(-2^{j}\right) \int_{B_{j}}\left|g_{0} \nu^{m}\left(\partial_{\tau} \Phi_{j}(\zeta) \partial_{\tau} \bar{\Phi}(\zeta)+\partial_{\nu} \Phi_{j}(\zeta) \partial_{\nu} \bar{\Phi}(\zeta)\right)\right| d \zeta
\end{aligned}
$$

since

$$
|\omega(\zeta)| \leq C \nu \leq C^{\prime} \nu\left|J_{\varphi}\right| \leq C^{\prime \prime} \exp \left(-2^{j}\right)\left|J_{\varphi}\right|
$$

on the sets $B_{j}$. From the expression (3.1) we see that

if

$$
1 \leq \frac{2}{\left|\partial_{\tau} \varphi(\zeta)\right|}
$$

$$
|\nu| \leq \min \left\{1,\left.\sup _{\tau \in \mathbf{R}}\left|2 \alpha_{2}^{\prime}(\tau) \alpha_{1}^{\prime \prime}(\tau) \alpha_{1}^{\prime}(\tau) \alpha_{2}^{\prime \prime}(\tau)+\right| \alpha^{\prime \prime}(\tau)\right|^{2} \mid\right\} .
$$

Hence, since $\nu^{m} \asymp h$ on $V$, it follows that

$$
\left|I^{\circ \circ}\right| \leq C \exp \left(-2^{j}\right) \int_{B_{j}} h(\zeta)\left(\frac{\left|\partial_{\tau} \Phi_{j}(\zeta) \partial_{\tau} \bar{\Phi}(\zeta)\right|}{\left|\partial_{\tau} \varphi(\zeta)\right|^{2}}+\left|\partial_{\nu} \Phi_{j}(\zeta) \partial_{\nu} \bar{\Phi}(\zeta)\right|\right)\left|J_{\varphi}\right| d \zeta,
$$

for all large enough values of $j$; for which (3.36) holds on $B_{j}$. Now by using the Cauchy-Schwartz inequality with respect to weighted $L^{2}$-norm

$$
\left\|f ; L^{2}\left(B_{j} ; w_{2}\right)\right\|:=\left(\int_{B_{j}}|f(\zeta)|^{2} w_{2}(\zeta) d \zeta\right)^{1 / 2}, \quad w_{2}(\zeta):=h(\zeta)\left|J_{\varphi}\right|,
$$

and the Lemma 3.32 similar way as in $I^{\prime \prime}$ estimate, we obtain 


$$
\left|I^{\circ \circ}\right| \leq C \exp \left(-2^{j}\right)\left\|\nabla_{x} \Psi_{j} ; L^{2}(\Omega)\right\|\left\|\nabla_{x} \Psi ; L^{2}(\Omega)\right\| \leq C \exp \left(-2^{j}\right),
$$

for all large enough values of $j$. Since $\left\|\Psi_{j} ; \mathcal{H}(\Omega ; \Lambda)\right\| \asymp 1$ and $\|\Psi ; \mathcal{H}(\Omega ; \Lambda)\|=1$. This shows that $I^{\prime}$ tends to zero as $j \rightarrow \infty$.

Same way as in (3.34), we have

$$
I^{-}=\int_{B_{j}} \Phi_{j}(\zeta) \bar{\Phi}(\zeta) d \zeta+\int_{B_{j}} \Phi_{j}(\zeta) \bar{\Phi}(\zeta) \omega(\zeta) d \zeta=: I^{\bullet}+I^{\bullet \bullet}
$$

and similar estimation as in (3.35) shows that

$$
\left|I^{\bullet \bullet}\right| \leq C \exp \left(-2^{j}\right) .
$$

Thus $I^{\bullet \bullet} \rightarrow 0$ as $j \rightarrow \infty$.

We are left with the term $I^{\circ}-\lambda I^{\bullet}$. Now integration by parts yields

$$
I^{\circ}-\lambda I^{\bullet}=\int_{B_{j}}\left(-\nabla_{\zeta} \cdot g_{0} \nu^{m} \nabla_{\zeta} \Phi_{j}(\zeta)\right) \bar{\Phi}(\zeta) d \zeta-\lambda \int_{B_{j}} \Phi_{j}(\zeta) \bar{\Phi}(\zeta) d \zeta .
$$

Furthermore, since

$$
\begin{aligned}
\partial_{\nu}\left(g_{0} \nu^{m} \partial_{\nu} \Phi_{j}(\zeta)\right)= & a_{j}\left(\partial_{\nu}\left(g_{0} \nu^{m} \partial_{\nu} U(\nu)\right) X_{j}(\zeta)+m g_{0} \nu^{m-1} U(\nu) \partial_{\nu} X_{j}(\zeta)\right. \\
& \left.+g_{0} \nu^{m} U^{\prime \prime}(\nu) X_{j}(\zeta)+g_{0} \nu^{m} U^{\prime}(\nu) \partial_{\nu} X_{j}(\zeta)+g_{0} \nu^{m} U(\nu) \partial_{\nu}^{2} X_{j}(\zeta)\right) \\
= & : a_{j}\left(Y_{j}^{0}(\zeta)+Y_{j}^{1}(\zeta)+Y_{j}^{2}(\zeta)+Y_{j}^{3}(\zeta)\right)
\end{aligned}
$$

and

$$
\partial_{\tau}\left(g_{0} \nu^{m} \partial_{\tau} \Phi_{j}(\zeta)\right)=a_{j} g_{0} \nu^{m} U(\nu) \partial_{\tau}^{2} X_{j}(\zeta)=: a_{j} Z_{j}(\zeta),
$$

we obtain, by using the identity (3.13), that

$$
I^{\circ}-\lambda I^{\bullet}=a_{j}\left(\int_{B_{j}}\left(W_{j}(\zeta), \bar{\Phi}(\zeta)\right) d \zeta+\int_{B_{j}}\left(Z_{j}(\zeta), \bar{\Phi}(\zeta)\right) d \zeta\right)
$$

where

$$
W_{j}(\zeta):=Y_{j}^{1}(\zeta)+Y_{j}^{2}(\zeta)+Y_{j}^{3}(\zeta)+i \frac{m-2}{2} \sqrt{\lambda g_{0}^{-1}} g_{0} \nu^{m / 2-1} U(\nu) X_{j}(\zeta)
$$

Now (3.10), (3.11), (3.12) and the estimates (3.15) imply

$$
\left|Y_{j}^{1}(\zeta)\right| \leq C \nu^{m-2}, \quad\left|Y_{j}^{2}(\zeta)\right| \leq C \nu^{m / 2-1}, \quad\left|Y_{j}^{3}(\zeta)\right| \leq C \nu^{m-2}
$$

for all $j$. Thus, since $m / 2-1<m-2$ and $0<\nu<1$ on $V$, we have

$$
\left|W_{j}(\zeta)\right| \leq C \nu^{m / 2-1} \quad \text { for all } j .
$$

Also, by use of (3.10) and (3.15), we see that

$$
\left|Z_{j}(\zeta)\right| \leq C \nu^{m}|\tau|^{-2} \text { for all } j,
$$

where $\operatorname{supp}\left(Z_{j}\right) \subset B_{j} \backslash D_{j}$. Hence, by applying the Cauchy-Schwartz inequality to both terms of (3.37) separately, yields

$$
\left|I^{\circ}-\lambda I^{\bullet}\right| \leq C a_{j}\left(\left(\int_{B_{j}} \nu^{m-2} d \zeta\right)^{1 / 2}+\left(\int_{B_{j} \backslash D_{j}} \nu^{2 m}|\tau|^{-4} d \zeta\right)^{1 / 2}\right)\left\|\bar{\Phi} ; L^{2}(V)\right\|,
$$


Now

$$
\begin{aligned}
\int_{B_{j}} \nu^{m-2} d \zeta & =\int_{\exp \left(-2^{j+1}\right)}^{\exp \left(-2^{j}\right)} \nu^{m-2} d \nu \int_{-\kappa \exp \left(-2^{j}\right)}^{+\kappa \exp \left(-2^{j}\right)} d \tau \\
& \leq C \exp \left(-(m-1) 2^{j}\right) \exp \left(-2^{j}\right)=C \exp \left(-m 2^{j}\right)
\end{aligned}
$$

and

$$
\begin{aligned}
\int_{B_{j} \backslash D_{j}} \nu^{2 m}|\tau|^{-4} d \zeta & =2 \int_{\exp \left(-2^{j+1}\right)}^{\exp \left(-2^{j}\right)} \nu^{2 m} d \nu \int_{+\kappa \exp \left(-2^{j}-1\right)}^{+\kappa \exp \left(-2^{j}\right)} \tau^{-4} d \tau \\
& \leq C \exp \left(-(2 m+1) 2^{j}\right) \exp \left(+3 \cdot 2^{j}\right)=C \exp \left(-(m-1) 2^{j+1}\right) .
\end{aligned}
$$

Substituting (3.22) in to (3.38) and using the estimates above, we get

$$
\begin{aligned}
\left|I^{\circ}-\lambda I^{\bullet}\right| & \leq C \exp \left(+2^{j}\right)\left(\exp \left(-m 2^{j-1}\right)+\exp \left(-(m-1) 2^{j}\right)\right) \\
& \leq C \exp \left(-(m / 2-1) 2^{j}\right) ;
\end{aligned}
$$

since $-m 2^{j-1}>-(m-1) 2^{j}$ for all $j$ because $m>2$, and

$$
\begin{aligned}
\left\|\bar{\Phi} ; L^{2}(\mathcal{V})\right\| & =\left(\int_{V}|\bar{\Phi}(\zeta)|^{2} d \zeta\right)^{1 / 2} \leq C\left(\int_{V}|\bar{\Phi}(\zeta)|^{2}\left|J_{\varphi}\right| d \zeta\right)^{1 / 2} \\
& =C\left(\int_{\mathcal{V}}|\bar{\Psi}(y)|^{2} d y\right)^{1 / 2}=C\left\|\bar{\Psi} ; L^{2}(\mathcal{V})\right\| \leq C^{\prime}\|\Psi ; \mathcal{H}(\Omega ; \Lambda)\| \leq C^{\prime}
\end{aligned}
$$

due to the inequality $1 \leq C\left|J_{\varphi}\right|$ and the Lemma 3.39 bellow. This yields that $I_{1} \rightarrow 0$ as $j \rightarrow \infty$, since $m>2$.

3.39. Lemma. The inequality

$$
\left\|\bar{\Psi} ; L^{2}(\mathcal{V})\right\| \leq C\|\Psi ; \mathcal{H}(\Omega ; \Lambda)\|
$$

is valid for all $\Psi \in \mathcal{H}(\Omega ; \Lambda)$.

Proof. We have

$$
\left\|\bar{\Psi} ; L^{2}(\mathcal{V})\right\| \leq\left\|\bar{\Psi}-\Psi(\cdot, 0) ; L^{2}(\mathcal{V})\right\|+\left\|\Psi(\cdot, 0) ; L^{2}(\mathcal{V})\right\| .
$$

By Cauchy-Schwartz

$$
\left\|\bar{\Psi}-\Psi(\cdot, 0) ; L^{2}(\mathcal{V})\right\| \leq\left\|\mathbf{h}^{1 / 2} ; L^{2}(\mathcal{V})\right\|\left\|\mathbf{h}^{-1 / 2}(\bar{\Psi}-\Psi(\cdot, 0)) ; L^{2}(\mathcal{V})\right\| .
$$

Hence the inequality (3.26) from the Lemma 3.25 gives

$$
\left\|\bar{\Psi} ; L^{2}(\mathcal{V})\right\| \leq C\left(\left\|\partial_{z} \Psi ; L^{2}(\mathfrak{V})\right\|+\left\|\Psi(\cdot, 0) ; L^{2}(\mathcal{V})\right\|\right) \leq C^{\prime}\|\Psi ; \mathcal{H}(\Omega ; \Lambda)\|
$$

3.7. Estimate for $\boldsymbol{I}_{0}$. First,

$$
\begin{aligned}
\left|I_{0}\right| & =\left|\left(\left(\mathbf{h}-g_{0} \nu^{m}\right) \nabla_{y} \Psi_{j}, \nabla_{y} \bar{\Psi}\right)_{V}\right| \\
& \leq \int_{\varphi\left(B_{j}\right)}\left|\mathbf{h}(y)-g_{0} \nu^{m}(y)\right|\left|\left(\nabla_{y} \Psi_{j}(y), \nabla_{y} \bar{\Psi}(y)\right)\right| d y \\
& \leq\left\|\mathbf{h}-\left.g_{0} \nu^{m}\right|^{1 / 2} \nabla_{y} \Psi_{j} ; L^{2}\left(\varphi\left(B_{j}\right)\right)\right\|\left\|\left|\mathbf{h}-g_{0} \nu^{m}\right|^{1 / 2} \nabla_{y} \bar{\Psi} ; L^{2}\left(\varphi\left(B_{j}\right)\right)\right\|
\end{aligned}
$$

by the Cauchy-Schwartz inequality. 
Now $\left|\mathbf{h}(y)-g_{0} \nu^{m}(y)\right| \leq C \nu^{m+1 / 2}(y)$ on the set $\mathcal{V}$, and since $\mathbf{h} \asymp \nu^{m}$ on $\mathcal{V}$, it follows that

$$
\left|\mathbf{h}(y)-g_{0} \nu^{m}(y)\right| \leq C \nu^{1 / 2}(y) \mathbf{h}(y) \quad \text { on the set } \mathcal{V}
$$

so

$$
\left\|\left|\mathbf{h}-g_{0} \nu^{m}\right|^{1 / 2} \nabla_{y} \Psi_{j} ; L^{2}\left(\varphi\left(B_{j}\right)\right)\right\| \leq C \exp \left(-2^{j-2}\right)\left\|\mathbf{h}^{1 / 2} \nabla_{y} \Psi_{j} ; L^{2}(\mathcal{V})\right\|
$$

and

$$
\left\|\left|\mathbf{h}-g_{0} \nu^{m}\right|^{1 / 2} \nabla_{y} \bar{\Psi} ; L^{2}\left(\varphi\left(B_{j}\right)\right)\right\| \leq C \exp \left(-2^{j-2}\right)\left\|\mathbf{h}^{1 / 2} \nabla_{y} \bar{\Psi} ; L^{2}(\mathcal{V})\right\|,
$$

since $\nu(y) \leq C \exp \left(-2^{j}\right)$ on $\varphi\left(B_{j}\right)$.

Now, since $\left\|\mathbf{h}^{1 / 2} \nabla_{y} \Psi_{j} ; L^{2}(\mathcal{V})\right\|=\left\|\nabla_{x} \Psi_{j} ; L^{2}(\Omega)\right\|$ and the lemma 3.32 holds for all $\Psi \in \mathcal{H}(\Omega ; \Lambda)$, we get

$$
\left|I_{0}\right| \leq C \exp \left(-2^{j-2}\right)\left\|\nabla_{x} \Psi_{j} ; L^{2}(\Omega)\right\| \exp \left(-2^{j-2}\right)\left\|\nabla_{x} \Psi ; L^{2}(\Omega)\right\| \leq C^{\prime} \exp \left(-2^{j-1}\right),
$$

since $\left\|\Psi_{j} ; \mathcal{H}(\Omega ; \Lambda)\right\| \asymp 1$ and $\|\Psi ; \mathcal{H}(\Omega ; \Lambda)\|=1$. Thus $I_{0} \rightarrow 0$ as $j \rightarrow \infty$.

\section{Remarks}

4.1. A sharper form of the previous results. In [8] a result is stated that interval $[c, \infty)$ is contained in the essential spectrum of the linearised water-wave problem, when $m=2$ and $c>0$ is a parameter depending on the geometry of the domain. A similar result for a domain with a submerged object touching the water surface at one point, is obtained in [16]. In both cases the Theorem 2.3 can be applied, so the results hold even in a sharper form. Namely, the intervals are in fact contained in the continuous spectrum of the problem.

4.2. Open problems. It is unknown whether or not there exists points in the point spectrum, which are berried inside the continuous spectrum, for the problem (1.2)-(1.4) when $m \geq 2$. In the linearised theory of water-waves these type of solutions are often called trapped modes.

Another open problem, concerning the spectrum of the problem (1.2)-(1.4), is that whether or not the lower bound for the continuous spectrum in the case $m=2$ (which was obtained in [8]) is optimal or not.

\section{References}

[1] Adams, R. A., and J. J. F. Fournier: Sobolev spaces, second edition. - Elsevier, Amstedam, 2003.

[2] Birman, M.S., and M.Z. Solomyak: Spectral theory of self-adjoint operators in Hilbert space. - Reidel Publishing Company, Dordrecht, 1986.

[3] Bonnet-Ben DhiA, A.-S., and P. Joly: Mathematical analysis of guided water-waves. SIAM J. Appl. Math. 53, 1993, 1507-1550.

[4] Gilbarg, D., and N. S. Trudinger: Elliptic partial differential equations of second order. Springer-Verlag, Berlin, 1977.

[5] Johnson, R. S.: A modern introduction to the mathematical theory of water waves. - Cambridge Texts Appl. Math., Cambridge Univ. Press, Cambridge, 1997.

[6] Kuznetsov, N., V. Mazya, and V. Vainberg: Linear water waves. A mathematical approach. - Cambridge Univ. Press, Cambridge, 2002. 
[7] Ladyzhenskaya, O. A.: Boundary value problems of mathematical physics. - Nauka, Moscow, 1973; English transl.: The boundary value problems of mathematical physics. Translated from the Russian by Jack Lohwater. - Appl. Math. Sci. 49, Springer-Verlag, New York, 1985.

[8] Martin, J., and J. TASKinen: Linear water-wave problem in a pond with shallow beach. Appl. Anal. (to appear).

[9] Nazarov, S. A.: A general scheme for averaging self-adjoint elliptic systems in multidimensional domains, including thin domains. - Algebra i Analiz 7:5, 1995, 1-92; English transl.: St. Petersburg Math. J. 7:5, 1996, 681-748.

[10] Nazarov, S. A.: Asymptotic theory of thin plates and rods. Volume 1. Dimension reduction and integral estimates. - Nauchnaya Kniga, Novosibirsk, 2001 (in Russian).

[11] Nazarov, S. A.: Concentration of the point spectrum on the continuous one in problems of linear water-wave theory. - Zap. Nauchn. Sem. S.-Peterburg. Otdel. Mat. Inst. Steklov. (POMI) 348, 2007, 98-126; English transl.: J. Math. Sci. (N. Y.) 152:5, 2008, 674-689.

[12] Nazarov, S. A.: On the spectrum of the Steklov problem in peak-shaped domains. - Tr. St.Peterbg. Mat. Obshch. 14, 2008, 103-168; English transl.: Trans. Amer. Math. Soc. (2) 14, 2009, 73-131.

[13] NazArov, S. A.: On the essential spectrum of boundary value problems for systems of differential equations in a bounded domain with a cusp. - Funktsional. Anal. i Prilozhen. 43, 2009, 55-67; English transl.: Funct. Anal. Appl. 43, 2009, 44-54.

[14] Nazarov, S. A., J. Sokolowski, and J. TASkinen: Neumann Laplacian on a domain with tangential components in the boundary. - Ann. Acad. Sci. Fenn. 34:1, 2009, 131-143.

[15] Nazarov, S. A., and J. Taskinen: On the spectrum of the Steklov problem in a domain with a peak. - Vestnik St. Petersburg Univ. Math. 41:4, 2008, 56-65.

[16] Nazarov, S.A., and J. Taskinen: On essential and continuous spectra of the linearized water-wave problem in a finite pond. - Math. Scand. 106:1, 2010, 141-160.

[17] Nazarov, S. A., and J. TASkinen: Radiation conditions at the top of a rotational cusp in the theory of water-waves. - Math. Model. Numer. Anal. 45:4, 2011, 947-979.

[18] Spiegel, M. R.: Schaum's outline of vector analysis and an introduction to tensor analysis, SI (metric) edition. - McGraw-Hill, New York, 1974.

[19] Stoker, J. J.: Water waves. The mathematical theory with applications. Reprint of the 1957 original. - Wiley Classics Library, John Wiley \& Sons, Inc., New York, 1992. 